\title{
Clinical assessment of interhemispheric psychological functioning ${ }^{1}$
}

\author{
J. G. BEAUMONT AND S. J. DIMOND
}

From the Department of Psychology, University College, Cardiff

SUMMARY A simple method by which the relative performance of the two cerebral hemispheres, and cooperation between the hemispheres, may be assessed in a clinical setting is discussed. The apparatus, which allows the findings of neuropsychological investigations of hemisphere performance in the normal and split-brain subject to be applied in the clinic, is described, together with the results of a preliminary trial and some clinical results.

With increasing interest in the lateral specialization of the human cerebral hemispheres, and in the results of commissurotomy and hemispherectomy with reference to clinical diagnosis and treatment in neurology and neurosurgery, there has been a realization of the potential of methods for the interhemispheric assessment of psychological functioning in the clinic. Investigation of the human split-brain (Gazzaniga, 1970) has revealed that each cerebral hemisphere has the capacity to perceive, to remember, and to learn, and that each hemisphere proceeds largely without awareness of the other. The 'split-brain' patient behaves in many ways as if he had two independent brains each with a mind of its own.

This approach to the analysis of brain function has been carried into the study of the normal human brain and in a variety of studies, using a divided visual field technique, the present authors have shown that the use of two hemispheres acts to increase total brain capacity in processing information (Dimond and Beaumont, 1971), that the right hemisphere has a greater proficiency in carrying out calculations on digits flashed to it (Dimond and Beaumont, 1972a), and that the right hemisphere is severely deficient in vigilance performance (Dimond and Beaumont, 1972b). Other findings support a conception of the left hemisphere as a language-oriented analytical system, and of the right hemisphere as a visuo-spatial apperceptive system.

1 This work was carried out under tenure of Grant G969/96C of the Medical Research Council.
The potential of an apparatus which could assess the relative functioning of each cerebral hemisphere, based on the divided visual field method and utilizing differences in the characteristics of the two hemispheres, and which would be used in the clinic for assessment, for diagnosis of cerebral lesions and even for rehabilitative adjustment of patients, is clear. An apparatus for this purpose and some preliminary results are described below.

\section{METHODS}

The apparatus, shown in Fig. 1, is of a size which will rest conveniently on a hospital bed-table and patients can be tested successfully while still confined to bed. The patient fixates a central pilot light and faces four numerical indicator tubes, two to the left and two to the right of fixation at $11^{\circ}$ and $22^{\circ}$ of visual angle. Two adjustable screens are arranged so that tube 1 projects to the nasal hemiretina of the left eye, tube 2 to the temporal hemiretina of the right eye, tube 3 to the temporal hemiretina of the left eye, and tube 4 to the nasal hemiretina of the right eye. Digits appearing at tubes 1 and 2 are therefore directed to the right hemisphere, and at tubes 3 and 4 to the left hemisphere. Stimuli directed to each hemisphere pass through both eyes so taking account of any differences in visual acuity between the eyes. The stimuli, digits $0-9$, may be presented at any source for a variety of exposure periods, but an exposure of $130 \mathrm{msec}$ has been employed by the present authors. This period is sufficiently short to prevent the distractible patient from reorienting his eyes from the point of fixation to the stimulus source. To allow for initial failure to fixate, the appearance 


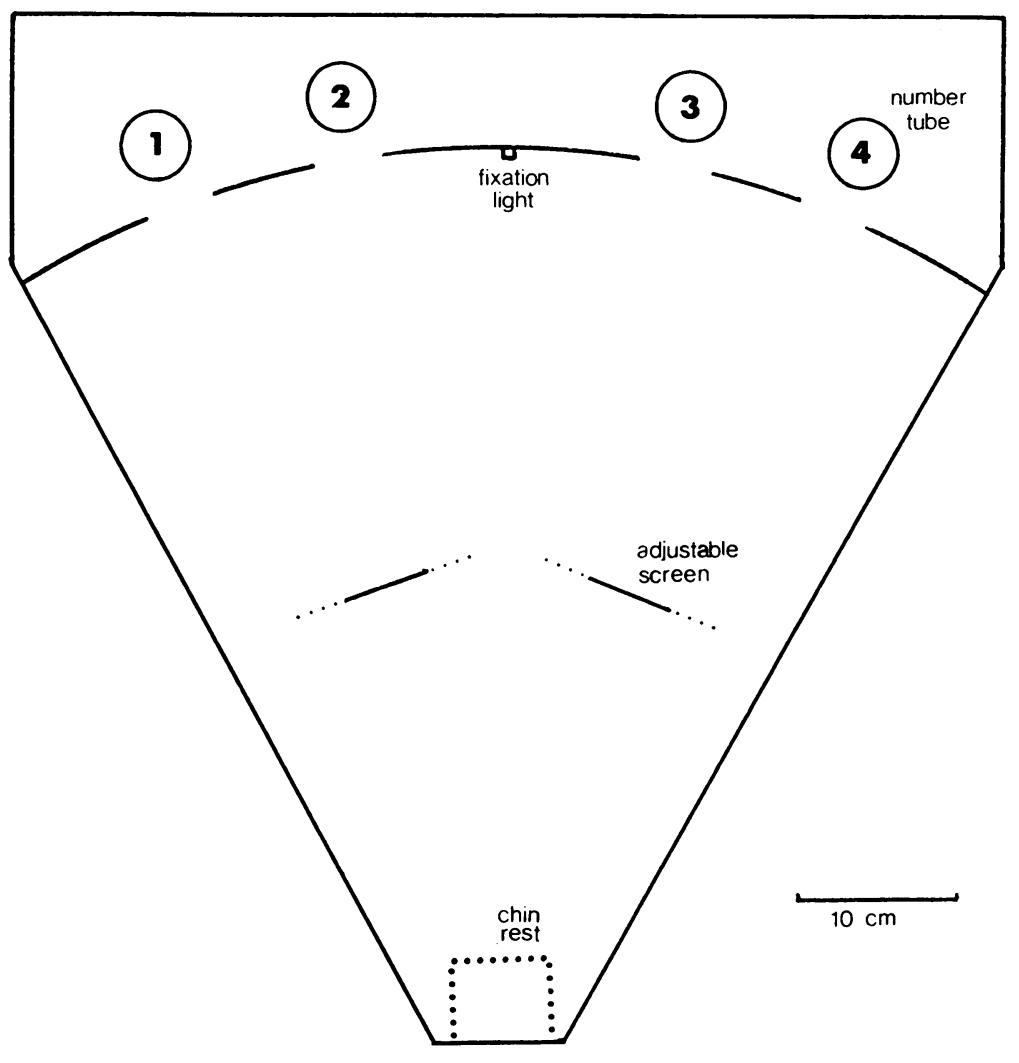

FIG. 1. Plan of the apparatus.

of stimuli at each source is randomized, so that, while the interhemispheric effect may be weakened, the overall logic of presentation cannot be disrupted. Facilities for the timing of response latencies are also incorporated and may be linked to a voice key, to a microswitch operated by either patient or examiner, or to any other transducer.

Stimuli are currently limited to the forms of indicator tube available, but the application of dot matrix displays allowing for non-symbolic stimulus presentation is under development. In preliminary trials, the authors have employed the numerical indicator tube and a battery of four tests: identifying a simple digit presented; matching a digit to auditory input; matching two digits presented simultaneously in the visual mode; searching for a digit given in the auditory mode among four presented in the visual mode.

The method, however, is naturally not limited to these particular tests, and the type of test which will prove of most value is a matter for empirical investigation.
The battery of tests described above was administered to a heterogeneous group of 24 neurological and neurosurgical patients, all of whom were considered to have unilateral cerebral lesions. Diagnoses were extremely varied ranging from transient cerebral ischaemia and carotid artery stenosis to cerebrovascular accident, fracture of the skull, and cerebral tumour. No patient with a visual field defect was included in the group as it was felt that the results of testing should, at this stage, reflect central psychological variables rather than peripheral perceptual effects.

An index of function was derived from the test battery, based on 16 indicator points, four from each test, and related to four criteria: hemisphere giving rise to fewer correct responses; hemisphere associated with more 'missed' stimuli; hemisphere yielding longer mean latency for correct responses; hemisphere yielding longer mean latency for total responses. This index, therefore, produces an indication of the relative functioning of each hemisphere and the likelihood of a pathological process within each hemisphere. 
RESULTS

Of the 24 patients tested, 12 showed an indicator difference between the hemispheres of two points or less, and these results are considered equivocal. Of the remaining 12 patients, $11(92 \%)$ showed an indicator difference which correctly predicted the side of lesion. There were no statistical differences between the results of patients with left and right lesions, and no single test appeared more valid than any other.

As a preliminary trial, this is seen to be strongly encouraging and work is proceeding to refine the battery and index of function in order to improve the prediction achieved. It is to be emphasized that the potential of the method is not considered to be dependent on these specific results, but four cases are briefly described below as an illustration of the procedure's validity.

\section{CASE 1}

A 42 year old man presenting with a 10 days' history of moderate right hemiplegia and slight dysphasia, was considered to suffer from left internal carotid artery stenosis, or possibly a cerebrovascular accident.

Total indicator points, left hemisphere $=11$

Total indicator points, right hemisphere $=1$

\section{CASE 2}

This 51 year old man, presenting with headaches, was seen postoperatively after the treatment of a large semicystic astrocytoma of the left frontal lobe.

Total indicator points, left hemisphere $=8$

Total indicator points, right hemisphere $=4$

\section{CASE 3}

This 40 year old man had seven days previously suffered a left frontal trauma with a $2 \times 2 \mathrm{~cm}$ fracture and slight laceration, although the dura mater was not broken.

Total indicator points, left hemisphere $=9$

Total indicator points, right hemisphere $=4$
CASE 4

A 50 year old man presenting with dyspraxia, limb pain, and astereognosis, was diagnosed to be suffering from transient cerebral ischaemia of the right hemisphere.

Total indicator points, left hemisphere $=4$

Total indicator points, right hemisphere $=8$

\section{DISCUSSION}

While it is clear that all psychological assessment procedures are only of value when seen in the context of the range of physical and clinical diagnostic trials available to the clinician, it is considered that growing knowledge of the organization of the brain must yield information which will be of value in the assessment and diagnosis of pathological states. The availability of a simple, cheap, and highly adaptable method of applying the results obtained in the laboratory in the clinical situation may lead to the development of a wide variety of tools which may benefit the clinician in a number of contexts.

This work was carried out under tenure of grant G969/96C of the MRC. We thank Dr. Spillane, Dr. Graham, Mr. Langmaid, and Mr. Weeks of the University Hospital of Wales for their help and cooperation, and Mr. Philip Kinsey for building the apparatus. Details of the construction of the equipment may be obtained from the authors.

\section{REFERENCES}

Dimond, S. J., and Beaumont, J. G. (1971). Use of two cerebral hemispheres to increase brain capacity. Nature, 232, 270-271.

Dimond, S. J., and Beaumont, J. G. (1972a). A right hemisphere basis for calculation in the human brain. Psychonomic Science, 26, 137-138.

Dimond, S. J., and Beaumont, J. G. (1972b). Vigilance: left hemisphere specialisation reflected in visual field differences. (In preparation.)

Gazzaniga, M. S. (1970). The Bisected Brain. Appleton: New York. 